\title{
Horizontal versus vertical wells interference in hydraulically fractured shale reservoirs
}

\author{
Samuel Igba, Lateef T Akanji, and Toochukwu Onwuliri \\ Petroleum Engineering Division, School of Engineering, University of Aberdeen, UK
}

Correspondence: Lateef Akanji, Petroleum Engineering Division, School of Engineering, University of Aberdeen, UK, Email l.akanji@abdn.ac.uk

Received: December 20, 2018 | Published: March 05, 2019

Copyright@ 2019 Igba et al. This is an open access article distributed under the terms of the Creative Commons Attribution License, which permits unrestricted use distribution, and reproduction in any medium, provided the original author and source are credited.

\begin{abstract}
The impact of well interference on in-situ stresses, drainage area, and pressure response in hydraulically fractured shale reservoirs is examined. In-situ stress distribution in the reservoir resulting from fracture propagation and poro-elasticity and its influence on hydraulic fracture orientation and well spacing configurations are studied using iterative numerical methods. The results of the simulation indicated that drainage distance $\left(\mathrm{X}_{\mathrm{DL}}\right)$ from the well centre is restricted to the immediate environment of the well and with little effect on the external reservoir. In contrast, in-situ stress change has a wider and more complex reservoir reach away from the well with stress orthogonal reorientation occurring from a distance-of-stress-orthogonality $\left(\mathrm{F}_{\sigma}\right)$, while pressure response has the farthest reach $\left(\mathrm{X}_{\mathrm{PT}}\right)$. A new approach utilised in this study, which considers in-situ stress, drainage area and pressure interference $\left(\right.$ such that $\left.\mathrm{X}_{\mathrm{DL}}<\mathrm{F}_{\sigma}<\mathrm{X}_{\mathrm{PT}}\right)$, suggests that a spacing range of $450 \mathrm{ft}$ to $750 \mathrm{ft}$, with an optimum of $600 \mathrm{ft}$ for minimal interference will be adequate. Furthermore, parallel orientation of infill wells within this range is less feasible due to complex stress reorientation over the productive years. Wells drilled and fractured perpendicular to the parent well showed incremental cumulative production.
\end{abstract}

Keywords: Well spacing, shale reservoir, interference, hydraulic fracturing

\section{Introduction}

Well interference induced by hydraulic fracturing can affect the development of shale reservoirs. Hydraulic fracture stimulation has been employed successfully to enhance production in very low permeability reservoirs that are in themicro or nano midrange (usually less than $1 \mathrm{md}$ ). Initially implemented on vertical wells, it was uneconomical to implement in low permeability shale reservoirs because of the high cost of drilling many vertical wells. ${ }^{1}$ Today, many reports have shown that the use of multistage hydraulically fractured horizontal wells proven to be a better option. Although, certain reservoir geometry and in-situ stress condition require vertical wells to be drilled; further advancement in this technology, specifically, in hydraulic fracturing design in horizontal wells and well spacing, increased its applicability in various shale plays.

Unlike fracture spacing optimisation, well spacing has no definite formula, but has been modelled to suit pressure profile and cumulative hydrocarbon output. In Eagle Ford shale, well spacing averages at $700 \mathrm{ft}$, but recently, it has become tighter. ${ }^{2}$ Reduced hydraulic fracture spacing with high fracture density and well density have been revealed to improverecovery. ${ }^{3-5}$ However, these studies have shown that closer well spacing in hydraulically fractured wells results in well-to-well interference. This may affect well performance overall development strategy.

In-situ stress, but not pressure, in the stress shadow and fracture drainage volume was the focus of earlier performance studies and resulted in the Zipper and Texas Two Step well and fracture patterns. In contrast, well spacing studies have centred on well drainage volume and pressure, but not in-situ stress. ${ }^{5}$ The implication of this is unfavourable hydraulic fracture stimulation of infill well leading to under optimised production. ${ }^{6}$ The modified zipper configuration attempted stress inclusion in its design to address this gap, but it is not widely implemented for interference reasons. ${ }^{7}$ The inclusion of in-situ stress distribution in addition to drainage volume and pressure transient will provide a holistic understanding of well-towell interference in shale reservoirs. This will help in placement and stimulation of wells

In this study, the effect of in-situ stresses and thermo-poroelasticity on production from shale reservoirs was investigated. A coupled geomechanics, heat and fluid flow model for hydrocarbon production in shale reservoirs is adopted. A simple reservoir model with two wells (one horizontal and one vertical)having several planar fractures was developed. We investigated drainage volume, pressure response and in-situ stress distribution of well interference. Sensitivity analysis was performed on the fracture orientation to determine the impact on fluid drainage. Our study provides key insights into well spacing and fracture orientation when developing infill wells.

\section{Model formulation}

The governing equations for hydrocarbon production in unconventional reservoirs include porous medium deformation, pore and fracture fluid flow, and fracture propagation. These equations are expressions of mass, momentum and energy equilibrium. Thermal energy changes due to production or injection have shown significant effect on in-situ stresses, thus heat has been accounted for in the model. ${ }^{8}$ 


\section{Geomechanical model}

Typical mechanical properties: Young's modulus (M), Shear modulus $(\mathrm{G})$, Poisson's ratio(v), Bulk Modulus (K) and Biot's coefficient $(\alpha)$, all makeup the fundamental component of a geomechanical model. Also, rock strength, which is the amount of resistance a body of rock must wedge against external energy, plays a significant role in fracture initiation and propagation. Unconfined compressive strength (UCS), Tensile strength $\left(T_{o}\right)$, cohesion $\left(C_{o}\right)$, and internal friction $(\theta)$, all make up different forms for rock strength that come into force to resist deformation in rocks

\section{Principal stresses}

These stresses play a major role in determining direction of wellbore during drilling and the direction of hydraulic fracture propagation. Zhao et al. ${ }^{9}$ demonstrated that hydraulic fracture reroutes its path towards the direction of maximum horizontal stress direction, irrespective of its direction of initiation. Implication of this travel path is the formation of simple non-planar fractures at early time with low fracture density, and complex fractures at late time with high fracture density. Thus, it is generally recommended that fracture stimulation be initiated in the direction perpendicular to the minimum horizontal stress.

Principal stresses acting on the subsurface; minimum and maximum horizontal stresses, are a translation of the effect of vertical stress (loading) at the depth of interest. Typical change in stress for anisotropic formation can be written as ${ }^{1}$ :

$$
\begin{gathered}
\sigma_{h}-\alpha_{h} P_{h}=\frac{E_{h}}{E_{v}} \frac{v_{v}}{1-v_{h}}\left[\sigma_{v}-\alpha_{v} P_{p}\right]+\frac{E_{h}}{1-v_{h}^{2}} \varepsilon_{H}+\frac{E_{h} v_{h}}{1-v_{h}^{2}} \varepsilon_{H} \\
\sigma_{H}-\alpha_{h} P_{p}=\frac{E_{h}}{E_{v}} \frac{v_{v}}{1-v_{h}}\left[\sigma_{v}-\alpha_{v} P_{p}\right]+\frac{E_{h}}{1-v_{h}^{2}} \varepsilon_{H}+\frac{E_{h} v_{h}}{1-v_{h}^{2}} \varepsilon_{h}
\end{gathered}
$$

where $\sigma_{h}$ and $\sigma_{H}$ are the minimum and maximum horizontal stresses respectively, $\alpha_{h}$ and $\alpha_{v}$ are Biot's horizontal and vertical coefficient respectively, $E_{h}$ and $E_{v}$ are Young Modulus in the horizontal and vertical direction respectively, $\varepsilon_{h}$ and $\varepsilon_{H}$ are minimum and maximum principal horizontal strain respectively, $v_{h}$ and $v_{v}$ are the horizontal and vertical Poison's ratio respectively.

\section{Porous medium deformation}

Porous medium deformation is modelled as a poroelastic material undergoing quasi-static deformation. At equilibrium, the initial stress in the medium is zero when body forces are neglected:

$$
\nabla . \sigma+F=0
$$

where, $\nabla . \quad$ is the divergence operator, $\sigma$ is the Cauchy total stress tensor and $F=\rho_{b} g$ is the body force, comprising of, $g$ the gravity vector and, $\rho_{b}$ the bulk density., $\rho_{b}=\phi \rho_{f}+(1-\phi) \rho_{s}$ where $\rho_{\text {is }}$ is fluid density, $\quad \rho$ is the density of the solid phase, and $\phi$ is the true porosity. The effective stress and rock constitutive relation for thermo-elasticity is given as ${ }^{10}$ :

$$
\begin{gathered}
\sigma=\sigma^{\prime}-\alpha \mathrm{p} 1, \\
\sigma^{\prime}=\mathrm{C}_{d r}: \varepsilon-3 \beta_{s} K_{d r} T 1,
\end{gathered}
$$

where, $\sigma^{\prime}$ is effective stress, $\alpha$ is the Biot's coefficient, $\beta$ is the coefficient of liner solid thermal expansion, $C_{d r}$ is the rank-4 drained elasticity tensor, $K_{d r}$ is the drained bulk modulus, 1 is the rank-2 identity tensor, $p \underset{d s}{i s}$ the pore pressure and $T$ is temperature. The incremental stress form that includes pore pressure and temperature effects for the geomechanical model can be written as:

$$
\sigma-\sigma_{0}=C_{d r}: \varepsilon-\alpha\left(p-p_{0}\right) 1-3 \beta_{s} K_{d r}\left(T-T_{0}\right) 1
$$

When subjected to small strain $\varepsilon$, the medium deforms to a new stress state:

$$
\nabla \cdot\left(C_{d r}: \varepsilon-\alpha p 1-3 \beta_{s} K_{d r} T 1\right)+\rho_{b} g=0
$$

The strain tensor, $\varepsilon$ due to the infinitesimal transformation assumption, is the symmetric gradient of displacement : $u$

$$
\varepsilon=\nabla^{s} u=\frac{1}{2}\left[\nabla u+(\nabla u)^{T}\right]
$$

and the resulting volumetric strain $\varepsilon_{v}$ is given as:

$$
\varepsilon_{v}=\varepsilon_{x x}+\varepsilon_{y y}+\varepsilon_{z z}=\nabla . u
$$

\section{Flow model}

For single-phase flow of a slightly compressible fluid in a poroelastic medium, the fluid mass conservation equation can be written as:

$$
\frac{\partial m_{n}}{\partial t}+\nabla . J_{\eta}=\rho_{f, 0} Q_{n}
$$

where, the accumulation term $\frac{\partial m_{\eta}}{\partial t}$ describes the time variation of fluid mass $(\eta=f)$ or heat energy $\quad(\eta=h)$ relative to the motion of the solid skeleton, $\quad J$ is the flux term, $\rho_{f, 0}$ is the reference fluid density and $Q_{n}$ is a source term. Darcy's and Fourier's laws are employed as conduction laws for fluid flow and heat flow.

\section{Pore fluid flow}

Under the assumption of small volumetric strains and Darcy flow, the thermoporoelasticity equation can be written by substituting Biot's poroelastic constitutive equation ${ }^{10}$ :

$$
\frac{1}{M} \frac{\partial p}{\partial t}+\alpha \frac{\partial(\nabla \cdot u)}{\partial t}-3 \beta_{m} \frac{\partial T}{\partial p}+\nabla \cdot\left(-\frac{k}{\mu}\left(\nabla p-\rho_{f} g\right)\right)=Q_{f}
$$

Where the velocity of fluid through the network of interconnected pores is given as:

$$
v_{i}=\frac{J_{n}}{\rho_{f, 0}}=-K_{f}\left(\nabla p-\rho_{f} g\right)
$$

Hydraulic conductivity $K_{f}=k / \mu$, where $k$ is permeability and $\mu$ is viscosity. $M$ is Biot modulus, $\beta_{m}$ is total (bulk) thermal expansion given as $\quad \beta_{m}=(\alpha-\phi) \beta_{s}+\phi \beta_{f}$ where $\beta_{f}$ is the fluid thermal expansion. $1 / M=\phi_{0} c_{f}+\left(\alpha-\phi_{0}\right) / K_{s}$ and $\quad \alpha=1-K_{d r} / K_{s}$ are coupling coefficients. $\quad c_{f}=1 / K_{f}$ is the fluid compressibility, $K_{f}$ 
is the fluid bulk modulus, and $K_{S}$ is the solid grain bulk modulus. The fluid flow equation can be written in terms of total compressibility , as employed in many traditional reservoir simulators

$$
\phi C_{t} \frac{\partial_{p}}{\partial t}-3 \beta_{m} \frac{\partial T}{\partial t}+\alpha \frac{\partial(\nabla \cdot u)}{\partial t}=\nabla \cdot\left(\frac{k}{\mu}\left(\nabla_{p}-\rho_{f} g\right)\right)
$$

\section{Geothermal effect}

In-situ temperature change during production or injection may account for additional change in in-situ stress. We have included reservoir temperature effect in the porous deformation and pore fluid flow equations above. The solution for stepwise change in temperature can be obtained by using Fourier's law as the heat conduction term and a thermoporoelastic constitutive equation ${ }^{10}$ in the conservation equation (Eq. 10). Thus, the thermal diffusion equation is obtained as ${ }^{8}$ :

$$
3 \beta_{s} K_{d r} \frac{\partial(\nabla \cdot u)}{\partial t}-3 \beta_{m} \frac{\partial p}{\partial t}+\frac{c_{d}}{T_{0}} \frac{\partial T}{\partial t}-\nabla \cdot\left(K_{h} \nabla T\right)=Q_{h}
$$

Where the total volumetric heat capacity is

$c_{d}=(1-\phi) \rho_{s} c_{s}+\phi \rho_{f} c_{f} . c . \quad c_{s}$ is skeleton volumetric heat capacity, $c$ is fluid volumetric heat capacity. The contribution from volumetric strain is small and usually neglected for computational convenience.

\section{Fracture initiation and propagation}

In response to change in stress, fracture propagation is modelled in accordance with linear elastic fracture mechanics (LEFM). ${ }^{11}$ The model predicts the amount of stress required to initiate and propagate fracture in terms of fracture geometry $(\mathrm{r},) \theta$ and stress intensity factor $\mathrm{K}_{\mathrm{I}}$.

$$
\sigma_{y y}=\frac{K_{I}}{\sqrt{2 \pi r}} \cos \frac{\theta}{2}\left(1-\sin \frac{\theta}{2} \sin \frac{3 \theta}{2}\right)
$$

$\mathrm{K}_{\mathrm{I}}$ is proportional to applied stress. The rock's maximum tensile strength corresponds to critical intensity factor, $\mathrm{K}_{\mathrm{IC}}$ (Fracture toughness) obtained at $r=0$ and $K_{I}$ dampens asymptotically with increasing $r$ until normal far field stress is achieved. At equilibrium, $\mathrm{K}_{\mathrm{IC}}$ is equal to applied intensity factor $\mathrm{K}_{\mathrm{I}}$. Thus, fracture propagation occurs when applied stress is greater than sum of the rock's maximum tensile strength and in-situ stress parallel to the direction of fracture.

Stress intensity at Equilibrium:

$$
\mathrm{K}_{\mathrm{I}}=\mathrm{K}_{\mathrm{IC}}
$$

Equation (15) and (16) represents initiation and propagation criteria of fractures. Additional information on the equations for the corresponding fracture height growth, width profile, fluid pressure and stress intensity factor can be found in Yang et al. ${ }^{12}$ and Weng et al. ${ }^{13}$

\section{Interference modelling}

The effect of communication of reservoir fluids between wells is significant. Developed models in this regard measure pressure effect which is directly impactful on fluid recovery in conventional reservoirs. This is not the case for shale reservoirs where fluid flow is primarily dependent on matrix permeability. Unconventional reservoirs may have low flow tendency, but interference is still possible for varying low permeability. ${ }^{14}$ Variable flow rate, amongst others, is characteristic of shale reservoirs and makes interpretation of well test difficult. Although, constant production rate is possible in vertical wells, it is uneconomical and inapplicable in gas well test. In horizontal wells, constant rate is practically impossible. ${ }^{15}$

Complexities of shale reservoirs influences fluid flow behaviour and accounts for varying production rates and pressure. The proposed correlation for multi-rate flow $Q_{n}$, and normalised pressure, $(\Delta P /$ $Q_{n}$ ) used to study and analyse production from shale formations was derived from instantaneous source function. The semi analytical model is presented in Appendix B. Pressure alone cannot determine well spacing in unconventional reservoirs because of its indirect impact on fluid flow. Thus, pressure interference in unconventional reservoirs would be assumed to occur in most cases. Consequently, interference as a result of other determinants like drainage volume and in-situ stress may be investigated and considered for optimum hydrocarbon recovery.

\section{Iterative solution approach using CMG-GEM}

In the iterative approach, the reservoir porosity $\Phi$, is first imputed from initial predetermined value, then subsequently updated by solving the thermoporoelasticity model:

$$
\Phi^{n+1}-\Phi^{n}=\left(c_{0}+c_{2} a_{1}\right)\left(p-p^{n}\right)+\left(c_{1}+c_{2} a_{2}\right)\left(T-T^{n}\right)
$$

The coefficients of reservoir porosity $c_{i}$ and $a_{j}$ are defined in appendix A

Pressure and saturation change is obtained from the fluid model, while reservoir porosity and its derivatives from the geomechanical model. The sequence of execution commences with validating initial reservoir properties at equilibrium. A dynamic process simulates pressure change due to production/injection and stress change due to pore pressure change (internal process). It also measures change in stress due to fracture stimulation. Porosity is updated, and the cycle is repeated. An adaptive implicit method in discretised system of fluid flow and geomechanical equations is used to model pressure.

\section{Model setup and verification}

The model is a synthetic shale system in a reservoir with dimensions $3600 \mathrm{ft}$ x $3600 \mathrm{ft} \times 100 \mathrm{ft}$ represented by a $24 \times 24 \times 1$ grid system and block length and width of $150 \mathrm{ft}^{2}$ each. The fluid system is atypical black oil system with low gas oil ratio (GOR) of Eagle Fordshale formation extracted from publicly available data contained in Table $1 \& 2$ obtained from Yu et al. ${ }^{16}$ and Simpson et al. ${ }^{17}$ (also Pilcher et al., unpublished data, 2017), and include GOR: 1,000 SCF/STB, and formation volume factor: $1.65 \mathrm{rb} / \mathrm{stb}$; values which were acertained at reservoir conditions in the run of Peng-Robinson equation of state.

Rock property contribution to flow is the relative permeability behaviour of the litho logy which is characteristic of cyclic and inter bedded organic-rich marl and limestone of Eagle Ford shown in Figures $3 \& 4$, was extracted from the report of Simpson et al. ${ }^{17}$ 
Table I Compositional data for the model. ${ }^{16}$

\begin{tabular}{lllllll}
\hline Component & $\begin{array}{l}\text { Molar } \\
\text { fraction }\end{array}$ & $\begin{array}{l}\text { Critical } \\
\text { pressure }\end{array}$ & $\begin{array}{l}\text { Critical } \\
\text { temperature }\end{array}$ & $\begin{array}{l}\text { Molar } \\
\text { weight }\end{array}$ & $\begin{array}{l}\text { Acentric } \\
\text { factor }\end{array}$ & $\begin{array}{l}\text { Parachor } \\
\text { coefficient }\end{array}$ \\
\hline & & $(\mathrm{atm})$ & $(\mathrm{K})$ & $(\mathrm{g} / \mathrm{gmol})$ & & \\
$\mathrm{CO}_{2}$ & 0.01821 & 72.80 & 304.20 & 44.01 & 02250 & 78.00 \\
$\mathrm{~N}_{2}-\mathrm{C}_{1}$ & 0.44626 & 45.24 & 189.67 & 16.21 & 0.0084 & 76.50 \\
$\mathrm{C}_{2}-\mathrm{C}_{5}$ & 0.17882 & 32.17 & 341.74 & 52.02 & 0.1723 & 171.07 \\
$\mathrm{C}_{6}-\mathrm{C}_{10}$ & 0.14843 & 24.51 & 488.58 & 103.01 & 0.2839 & 297.42 \\
$\mathrm{C}_{11+}$ & 0.20828 & 15.12 & 865.00 & 304.39 & 0.6716 & 661.45 \\
\hline
\end{tabular}

Table 2 Data for reservoir, well, hydraulic fracture, and geomechanics

\begin{tabular}{llll}
\hline Description & Value & Description & Value \\
\hline Initial reservoir pressure & $8,000 \mathrm{psi}$ & Fracture height & $\mathrm{I00} \mathrm{Ft}$ \\
Bubble point pressure & $3446 \mathrm{psi}$ & Fracture width & $0.0 \mathrm{I} \mathrm{Ft}$ \\
Reservoir temperature & $270{ }^{\circ} \mathrm{F}$ & Fracture permeability & $10,000 \mathrm{mD}$ \\
Reservoir permeability & $470 \mathrm{nD}$ & Number total fractures & 22 \\
Reservoir porosity & 0.12 & Poisson's Ratio & 0.26 \\
Initial water saturation & $17 \%$ & Young modulus & $\mathrm{I} .5 \times 10^{6} \mathrm{Psi}$ \\
Total compressibility & $3 \times 10^{-6} \mathrm{I} / \mathrm{psi}$ & Thermal coefficient & $2.778 \mathrm{e}-6 \mathrm{I} /{ }^{\circ} \mathrm{F}$ \\
Formation thickness & $100 \mathrm{ft}$ & Cohesion & $50 \mathrm{psi}$ \\
Oil gravity & $41{ }^{\circ} \mathrm{API}$ & Biot coefficient & $\mathrm{I}$ \\
well spacing & $300,450,600,750 \mathrm{Ft}$ & Rock compressibility & $3 \mathrm{e}-5 \mathrm{I} / \mathrm{psi}$ \\
well Length (Horizontal) & $3,300 \mathrm{Ft}$ & Overburden stress & $8074 \mathrm{psi}$ \\
Flow across boundary & $0 \mathrm{stb} / \mathrm{d}$ & Maximum horizontal stress & $5503 \mathrm{psi}$ \\
BHP & $3,200 \mathrm{psi}$ & Minimum horizontal stress & $5255 \mathrm{psi}$ \\
Production rate & $2000 \mathrm{Stb} / \mathrm{d}$ & Overburden stress gradient & $\mathrm{I} .05 \mathrm{Psi} / \mathrm{ft}$ \\
Fracture half-length & $225 \mathrm{Ft}$ & Maximum horizontal stress & $0.7 \mathrm{Psi} / \mathrm{ft}$ \\
Fracture conductivity & $100 \mathrm{Ft}$ & Friction angle & $20^{\circ}$ \\
\hline
\end{tabular}

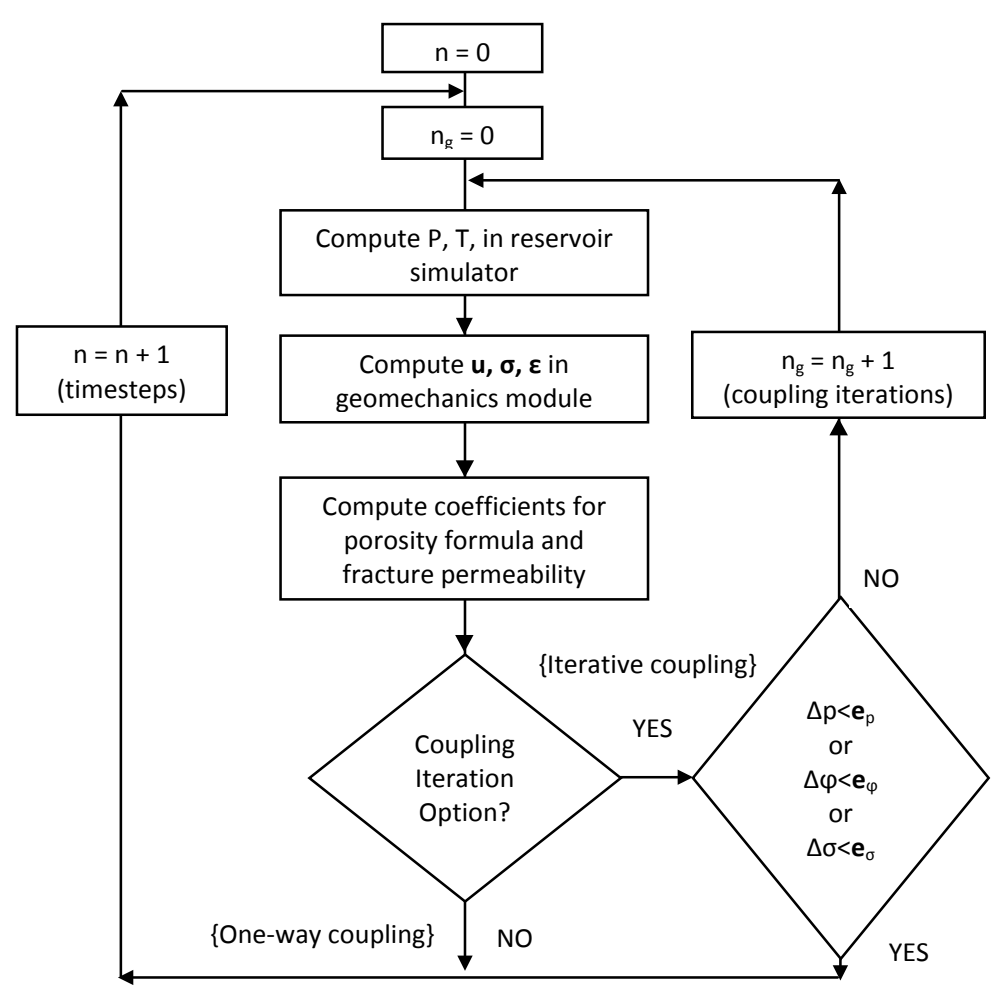

Figure I Iterative coupling approach (CMG-GEM) 


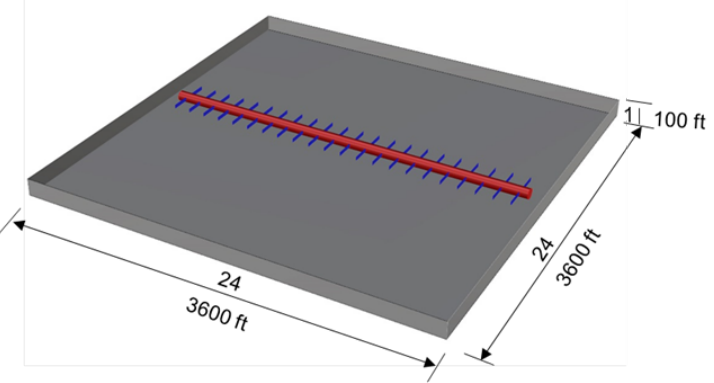

Figure 2 Conceptual reservoir model with $150 \mathrm{ft}$ fracture spacing

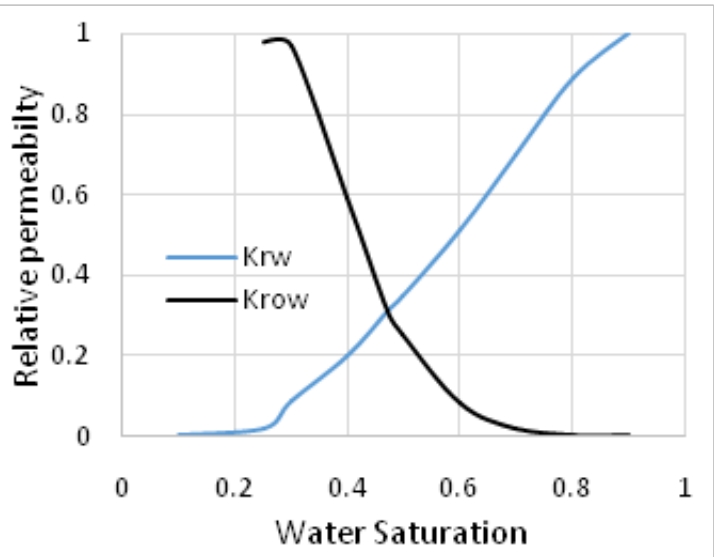

Figure 3 Water-Oil relative permeability curve

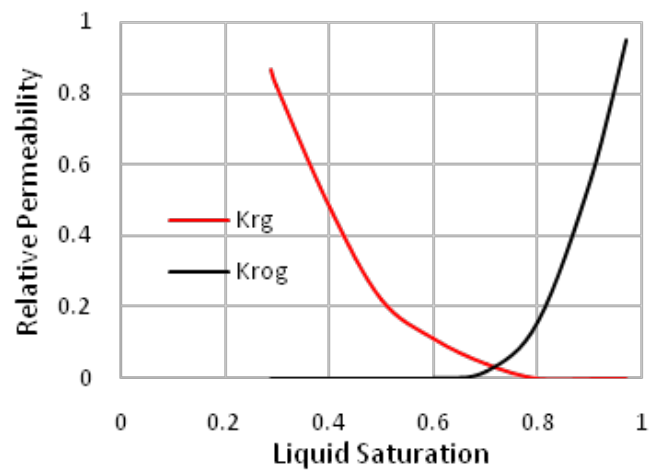

Figure 4 Liquid-gas relative permeability curve

\section{Strategy model-output focus}

In order to achieve our objectives, the following outcomes from the simulation were selected for investigation: Production rate and Cumulative production; Pressure and pressure drop (Field pressure, Bottom Hole Pressure, BHP); Oil saturation (Drainage volume per unit area); Minimum stress and Minimum principal effective stress vector; and, Maximum stress and Maximum principal effective stress vector. The usual output focus for interference and well spacing studies is production rate, cumulative production, and pressure. CMGGEM outputs drainage volume and principal stress vector, which is beneficial for an improved optimization study in unconventional reservoir.

\section{Results and discussions}

Oil production from tight sands has a characteristic steep drop in production rate after fluid in the immediate fracture is produced. Applying a minimum bottom-hole pressure of $3200 \mathrm{psi}$ and maximum stock tank oil rate of 2,000 BOPD at surface, Figures 5 and 6 show oil and gas rate respectively from horizontal well with 22 fractures at 150 fracture spacing.

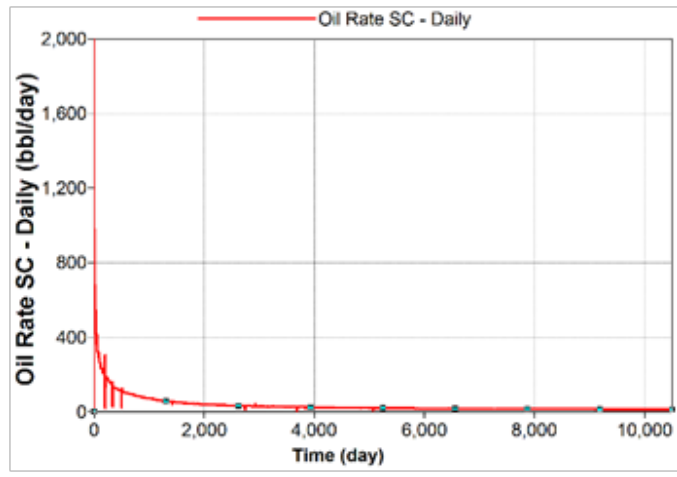

Figure 5 Oil production rate

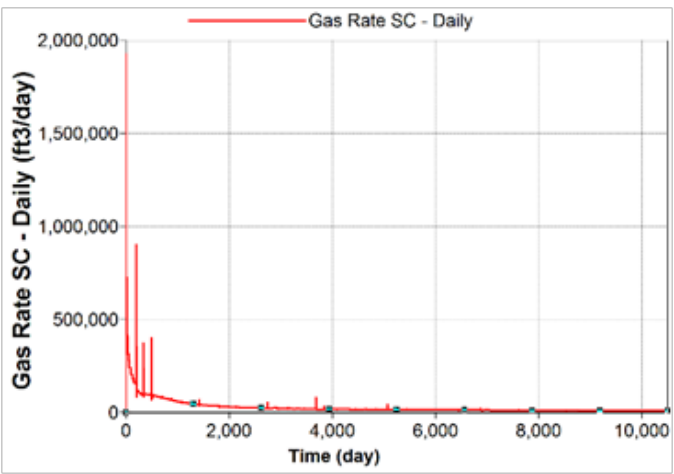

Figure 6 Gas production rate

Spikes seen in the rate are indicative of variable non-uniform production rates. At early times, fluid collected in the pores of the fractures within the stimulated reservoir volume (SRV) are produced in large volume. This is represented by high rates recorded until the stimulated volume is depleted. Further on, production depends on fluid transmissibility from matrix into fractures. More so, subsequent variable rates are due to the number and conductivities of secondary fractures present and generated in the process. Comparatively, a similar output for history matched model is shown in Figure 7.

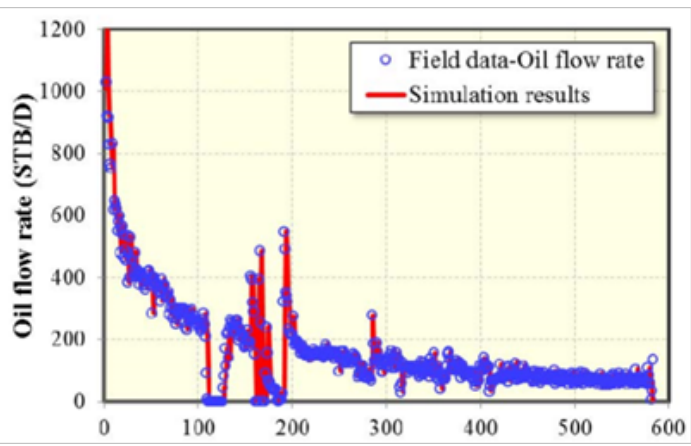

Figure 7 History-matched oil flow rate. ${ }^{18}$ 


\section{Case study}

To properly evaluate well placement and interference, three key physical properties are be examined for impact of the reservoir on regions away from the well location. This study examines drainage volume, pressure transient, and effective minimum principal stress.

\section{Drainage volume}

Single fracture - vertical well: The 2D simulation model for this test consists of a vertical well with planar fractures propagating outwards and penetrating total reservoir thickness. The drainage for a simulated productive period of 30 years is shown in Figure 8a assuming that the well is in a virgin pressure region. Drainage coverage stretches beyond the stimulated reservoir volume (SRV) by about $75 \mathrm{ft}$ over its fracture half length, and more than $150 \mathrm{ft}$ from the fracture face (west - east direction). Maximum volumetric output is possible without any form of nearby connection or interference. This is not expected in multi-fracture system. Instead, reduced fracture performance and far reaching effect into the reservoir.

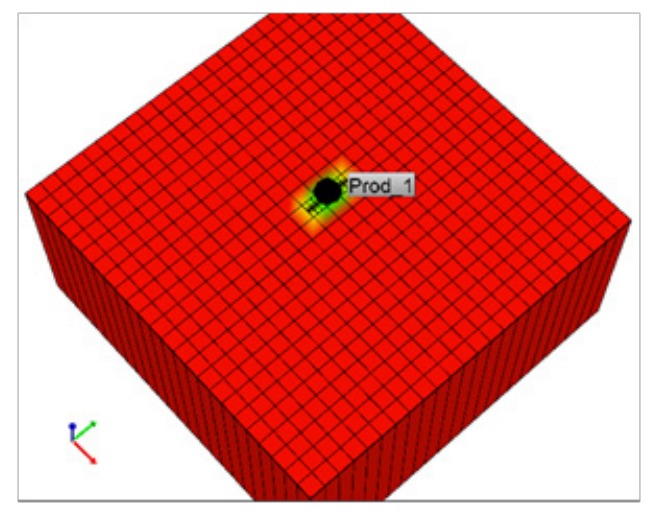

a. Vertical well

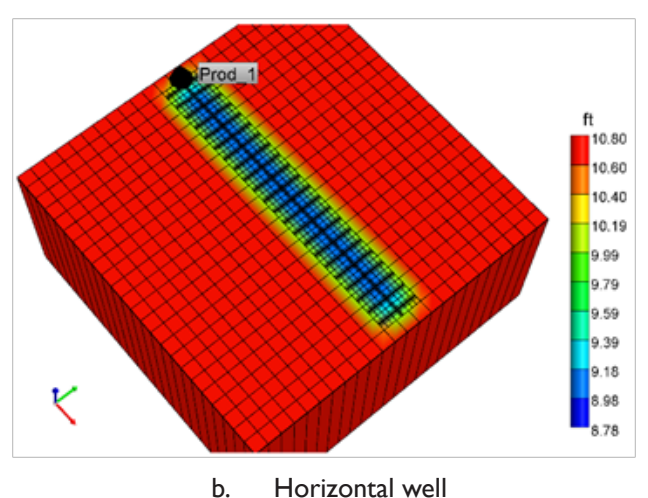

b. Horizontal well

Figure 8 Field oil drainage:volume per unit area (a) vertical well (b) horizontal well

Multiple fracture - horizontal well: In Figure 8b, a single horizontal well has more drainage coverage than the vertical well. This is because of its lateral length and number of fractures. Drainage advancement is consequent on merging of single regions by two or more fractures. The combined influence results in extended reservoir reach beyond fracture half length (a total distance $\mathrm{X}_{\mathrm{DL}}$ ); although, a little longer than seen in the vertical well. But Drainage length, at both ends of the well, opposite a fracture face, is the same as in single fracture vertical well. Basically, number of fractures is inversely proportional to fracture spacing. Table 3 shows drainage length gain beyond SRV for corresponding fracture spacing.
Table 3 Table of fracture data and drainage distance

\begin{tabular}{lllll}
\hline $\begin{array}{l}\text { Fracture } \\
\text { spacing }\end{array}$ & $\begin{array}{l}\text { Fracture } \\
\text { density }\end{array}$ & $\begin{array}{l}\text { Drainage } \\
\text { length, } \mathrm{X}_{\mathrm{DL}}\end{array}$ & $\begin{array}{l}\text { Half Fracture } \\
\text { length, } \mathrm{X}_{\mathrm{f}}\end{array}$ & $\begin{array}{l}\text { Extended length, } \\
\Delta \mathrm{X}_{\mathrm{DL}, \mathrm{f}}\end{array}$ \\
$\mathrm{ft}$ & $13300 \mathrm{ft}$ & $\mathrm{ft}$ & $\mathrm{ft}$ & $\mathrm{ft}$ \\
600 & 6 & 280 & 225 & 55 \\
450 & 8 & 280 & 225 & 55 \\
300 & 12 & 280 & 225 & 55 \\
150 & 22 & 340 & 225 & 115 \\
\hline
\end{tabular}

Table 4 Contrast in well configuration studies

\begin{tabular}{|c|c|}
\hline Existing studies & This Study \\
\hline $\begin{array}{l}\text { Pressure-only interference } \\
\text { (Typical of Conventional } \\
\text { reservoir) }\end{array}$ & $\begin{array}{l}\text { Considers interference in pressure, } \\
\text { as well as drainage area and in-situ } \\
\text { principal stresses. }\end{array}$ \\
\hline $\begin{array}{l}\text { Spacing of interfering infill well } \\
\text { is independent of production } \\
\text { effect of parent well. }\end{array}$ & $\begin{array}{l}\text { Infill well spacing and hydraulic } \\
\text { fracture orientation depends on } \\
\text { the duration of production of } \\
\text { parent well as it affects in-situ stress } \\
\text { orientation }\end{array}$ \\
\hline $\begin{array}{l}\text { Infill wells always have same } \\
\text { orientation with parent well }\end{array}$ & $\begin{array}{l}\text { This is achievable only if all the } \\
\text { wells are drilled and stimulated at } \\
\text { same time. } \\
\text { An infill well cannot have same } \\
\text { orientation with parent well beyond } \\
450 \mathrm{ft} \text { well spacing after years of } \\
\text { production. }\end{array}$ \\
\hline
\end{tabular}

Volumetric gain from the hydraulically fractured horizontal well is about $1400 \%$ more than that of the vertical well. This gain is subject to incremental number of fractures resulting from reduced fracture spacing shown in Figure 9. Overall production performance is good, but the volumetric performance of each fracture is less than recorded for vertical well.

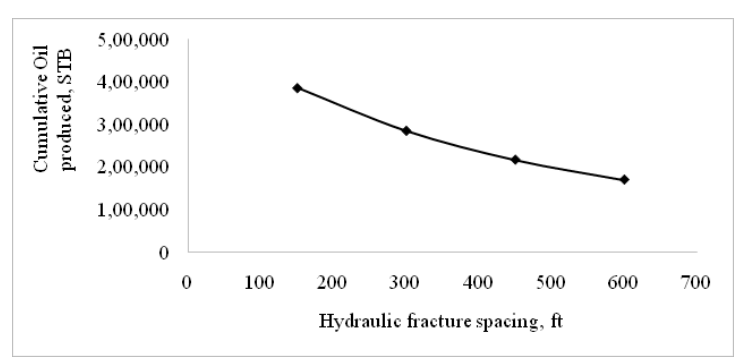

Figure 9 Cumulative Oil production against hydrauic fracture spacing

\section{Pressure transient}

Pressure drop at the well throughout the field life is up to $30 \%$ and $54 \%$ for the vertical and horizontal well respectively. But pressure at the outer reservoir remains unchanged at $8000 \mathrm{psig}$. Distance between these extreme pressures make up distance travelled by pressure transient disturbance $\left(\mathrm{X}_{\mathrm{PT}}\right)$ and connotes limit of interference in the adjacent well. In the single fracture vertical well, $\mathrm{X}_{\mathrm{PT}}$ is $470 \mathrm{ft}$.But in a multiple hydraulic fracture system, transient travel distance varies with fracture spacing as shown in Figure 10.

The lowest fracture spacing, $150 \mathrm{ft}$, accounts for maximum pressure transient travel of $640 \mathrm{ft}$. A distance marking minimum well spacing for limited interference effect. This distance, $\mathrm{X}_{\mathrm{PT}}$ is higher than the corresponding drainage length $\left(\mathrm{X}_{\mathrm{DL}}\right)$ for same fracture spacing, but 
less than $700 \mathrm{ft}$ well spacing commonly employed in practice. At maximum drainage length, $340 \mathrm{ft}$, corresponding pressure is $5614 \mathrm{psi}$ and is higher than BHP. The pressure gradient can still support production. Hence, drainage distance can be used as the yard stick for placing adjacent well for optimum reservoir drainage, but with pressure interference. In shared relation, both $\mathrm{X}_{\mathrm{DL}}$ and $\mathrm{X}_{\mathrm{pT}}$ are affected by fracture half length, and serve as a key determinant of well spacing. However, minimum stress is another criterion that can give a more meaningful conclusion since it affects fracture orientation and geometry.

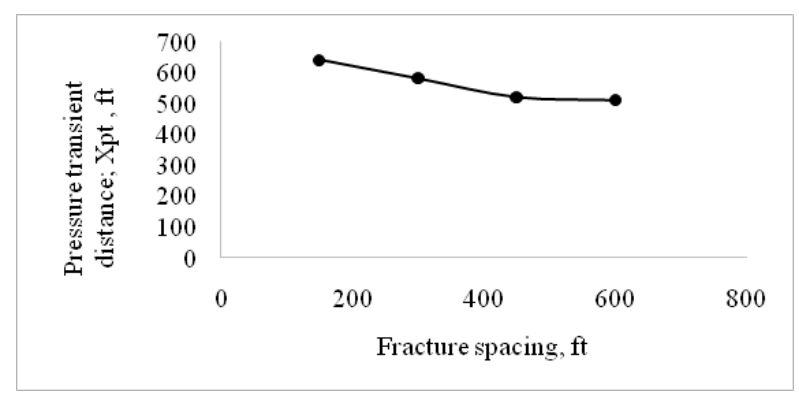

Figure 10 Pressure transient travel distance verus hydraulic fracture spacing

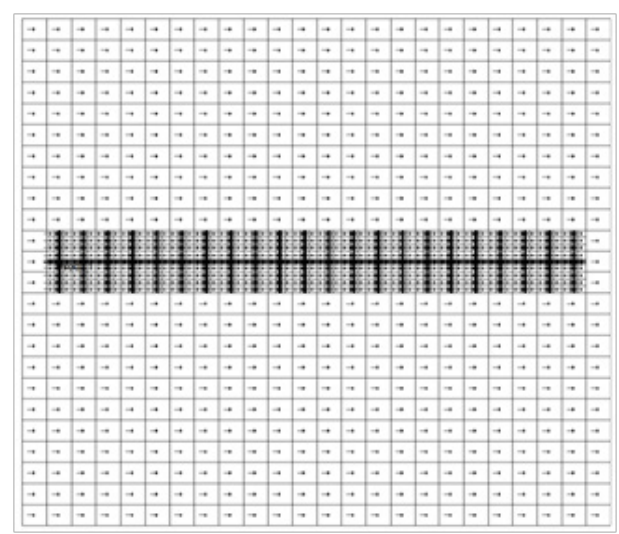

a. Stresses in $X Y$ plane

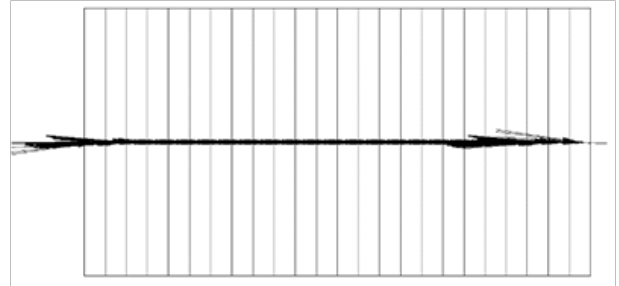

b. Stresses in $\mathrm{YZ}$ plane

Figure I I Minimum effective stress vector at initial time (CMG)

\section{In-situ stress distribution}

Minimum principal stress and its orientation play critical role in hydraulic fracture stimulation. At initial condition, principal stress is unidirectional, and its vector is shown in Figure11(a,b)with horizontal well running from left to right and hydraulic fractures propagating perpendicular to the minimum principal stress in the north-south direction. Altered by mechanical (hydraulic fracture) and poro-elastic effects (pore pressure changes), the stress vector changes direction from $0^{\circ}$ to $90^{\circ}$ as its magnitude changes with defined gradient.
Separated by 'axes-of-rotation' running horizontally through fracture/ well centre and perpendicular through the centre of fracture/mid-length of horizontal well, two kinds of stresses can be defined. Backslash ( ), where North-West and South-East stresses rotate anticlockwise, and Forward slash (/), where North-East and South-West stress rotate clock wise. At maximum rotation, minimum effective principal stress changes orientation to vertical direction pointing southward. Around the fractures, opposite the fracture faces, stress rotates in response to fracture opening and they rotate more during production.

Due to fluid continuity, sustained production alters pressure further away from the well, consequently, causing stress rotation between the wells and a maximum distance into the reservoir (far-field). Figure 12 shows stress response to vertical and horizontal wells. In both wells, only stress vectors acting along axes-of-rotation are aligned in extreme rotation $\left(0^{\circ}\right.$ and $\left.90^{\circ}\right)$ for all distance from the well into farfield reservoir while others stress vectors take any acute inclination.

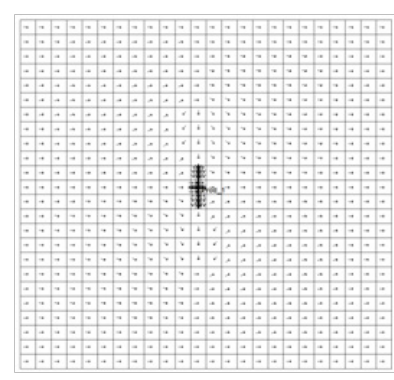

a.Vertical well (XY plane)

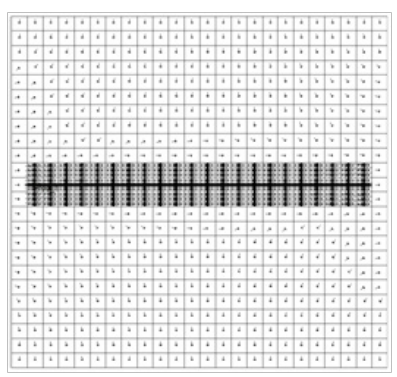

b. Horizontal well (XY plane)

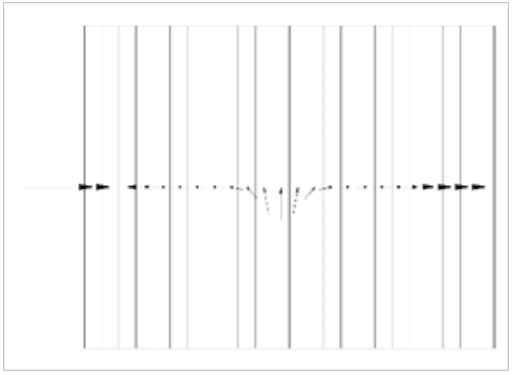

c. Vertical well (YZplane)

Figure 12 Minimum effective principal stress reorientation around (a) vertical well, (b) horizontal well and (c) YZ view, at $5^{\text {th }}$ year of production (CMG)

Furthermore, a different peculiar pattern was observed in horizontal well. Stress acting in the direction of fracture face, over its full length, align in horizontal direction $\left(0^{\circ}\right)$ after a short period of production. The complex orientation of stresses seen around fracture at start of production all tend toward horizontal alignment over the observed time. The initial distortion in stress could be attributed to transient response of stress to transition from static to dynamic flow state, whereas the stress alignment afterwards could be attributed to a steady flow condition. This horizontal tendency in stress vectors, spreads beyond the fracture length (or SRV) into external reservoir at distance of stress orthogonality $\mathrm{F}_{\sigma}$, measured from horizontal axis of the well. Beyond this distance, vertical acting stress vectors become dominant. At first, vertical oriented stress vectors act along the vertical axis of horizontal well, subsequently, this pattern spreads to the left and to the right simultaneously as production continues. At very long time, the whole stress vectors align in such a pattern that makes the 
whole horizontal well's length act like a single fracture as seen in vertical well. In both wells, orthogonal stress vectors along the axis of rotation assume their extreme anglesas production commences, while surrounding vectors take form later.

\section{Stress orthogonality}

In vertical well, two distances of stress orthogonality $\left(\mathrm{F}_{\mathrm{\sigma}}\right)$ can be seen; one at the centre of the well $\left(\mathrm{F}_{\sigma \mathrm{\sigma}}\right)$ with zero magnitude and the second $\left(\mathrm{F}_{\sigma 2}\right)$ at $750 \mathrm{ft}$. In horizontal well, $\mathrm{F}_{\sigma 1}$ measured from centre of the well varies with fracture spacing by observation. Figure 13 shows this relationship. This behaviour is most likely the effect of combination of stress characteristics of individual fractures. The early time post production horizontally aligned stresses take shape within $\mathrm{F}_{\sigma 1 .}$ However, $\mathrm{F}_{\sigma 2}$ could not be determined due to the size of the model. Even if made visible by increasing the model, the magnitude is large enough to validate well placement conclusions as reported in this study.

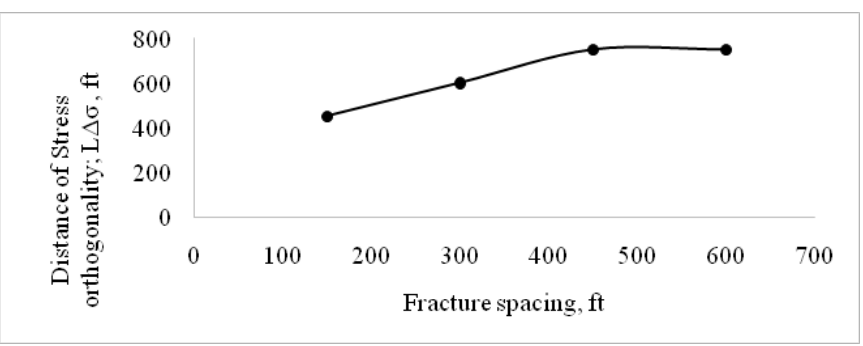

Figure 13 Distance of stress orthonality versus fractue spacing

Following the previous discussion, the model with lowest fracture spacing (150ft) gave highest production and corresponds to $\mathrm{F}_{\sigma 1}$ of $450 \mathrm{ft}$. Though, at very late time, this distance increases to $600 \mathrm{ft}$. In summary, by combining and comparing the effect of drainage, pressure and stress on near and far field reservoir, $\mathrm{X}_{\mathrm{DL}}$ is less than $\mathrm{F}_{\sigma}$, and both are less than $\mathrm{X}_{\mathrm{PT}}$ in the relation:

$$
X_{D L}<t_{\sigma 1}<X_{P T}
$$

This correlation constitutes recommended region of placement of in fill well following period of production from parent wells. In very low permeability reservoir, it is very tempting to assume placement of infill well outside the stimulated drainage region $\mathrm{X}_{\mathrm{DL}}$. The consequence would be poor hydraulic fracture job with high pressure interference. However, placing the well beyond $\mathrm{F}_{\sigma 2}$ could be the better guess. This is because it leaves pressure interference effect to latter time when a good portion of the reservoir must have been produced.

\section{Infill model: new well placement}

To optimise the suggested region of well placement for minimal interference, the first infill model was a vertical well located at $450 \mathrm{ft}$ next to the parent horizontal well with 22 hydraulic fractures at $150 \mathrm{ft}$ fracture spacing. Hydraulic fractures of both wells are parallel. The resulting drainage, stress, and pressure profile are shown in Figures 14 and 15. Hypothetically, the vertical well can be placed as such and yet have good stimulation, although complex northward effect of fracture hit is expected to take prominence. Nonetheless, hydrocarbon output would determine the effectiveness of the idea.

\section{Drainage profile}

Overall, there seems to be a merger in drainage volume per unit area as shown in Figure 14. Drainage length in the horizontal well closely matches its single state, but that of the vertical well shows less drainage compared to its standalone state (Figure 8a). The effect is evident in cumulative oil production by a reduction of $2 \%$. This is a result of drainage area interference which is consequent on created fracture network and fracture hit. The region of intersection of both fractures is highly depleted, probably the positive effect of fracture hit. The overall output reduction could be because of direction of flow of the reservoir fluids; away from the vertical well and towards the horizontal well.

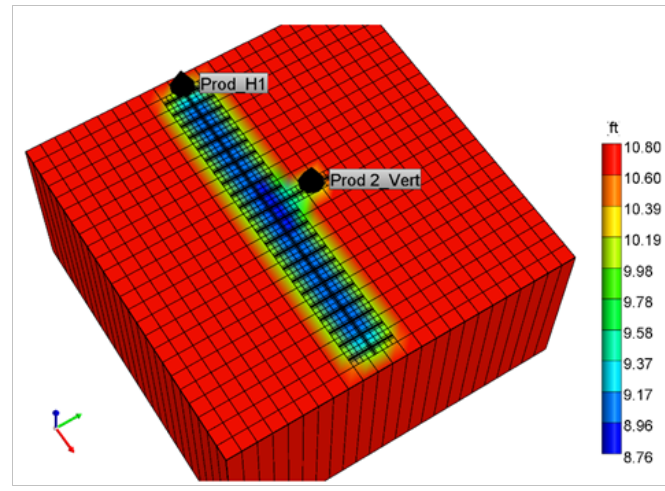

Figure I 4 Drainage volume per unite area of vertica and horinatal wells

\section{Stress and field pressure}

The merger effect is demonstrated in the distribution of minimum effective principal stress vector as in Figure 15. The Far field stress vectors are in conformity with standalone horizontal well. Stress vector around the vertical well has fair semblance with its standalone profile. The combined stress vector act like a single horizontal well. However, difference lies in the distance of orthogonality. Asymmetric in the horizontal well, $\mathrm{F}_{\sigma 1}$ increased in the north by $150 \mathrm{ft}$ but remained zero in the vertical well. Because of the location of both wells, their vertical axis aligns into a single vertical axis. It is expected that if the vertical well is relocated further left or right of its current position, the overall effect will be a complete spread of vertical orientation of minimum effective principal stress over the length of the horizontal well above $\mathrm{H}_{\sigma 1}$.

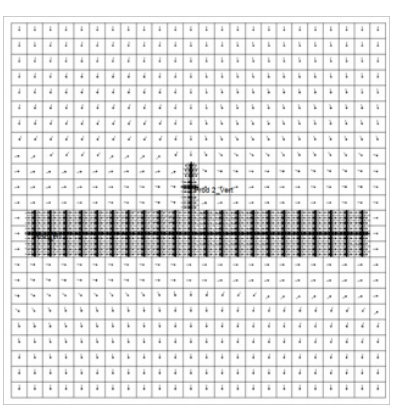

(a) Stress vector

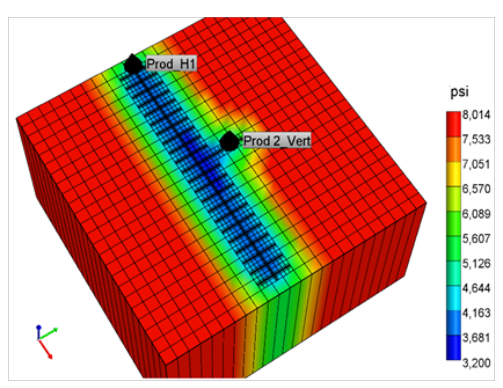

(b) Field Pressure
Figure 15 (a) Minimun effective principal stres vector, and (b) Field pressure for interfering wells

\section{Reverse infill model}

In this model, the infill vertical well was located within an area where parent horizontal well has influence. The objective was to locate the infill well within a region of horizontal oriented minimum effective principal stress so that vertical plan arhydraulic fractures can be 
achieved. The consequence was interference resulting in drainage area and pressure. In reverse, horizontal infill well located close to a vertical well within same spacing is possible. But absolute transverse fractures could not be achieved on its north side due to altered stress state around the vertical well. Non-planar and complex fractures resulted. Overall drainage is not expected to be affected and stress response to the horizontal infill well still dominates at later time. To avoid early conclusion on this interference effect, this study examined other possible well spacing, where optimum fracture propagation could be achieved.

\section{Sensitivity}

To evaluate interference against well spacing within permissible range expressed by equation (18), the distance between $450 \mathrm{ft}$ and $750 \mathrm{ft}$ was demarcated for parallel vertical fractures, and $300 \mathrm{ft}$ to $750 \mathrm{ft}$ for horizontal fractures in the vertical infill well.

\section{Horizontal and vertical wells - parallel fracture}

Interference is a function of well spacing. At wider well spacing, drainage area interference is absent. Closer well spacing results in increased interference. In Figure 16a, drainage section is independent for each well. But, in b and c convergence builds due to some central tendency harbouring potential force. Pressure, which travels farthest at the boundary front, is the potential causing this interference. Volumetric implication is seen in Figure 17.

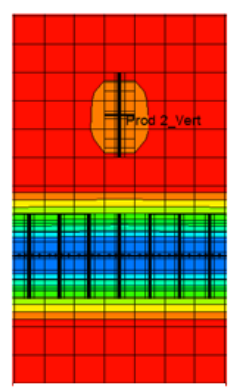

a. $750 \mathrm{ft}$

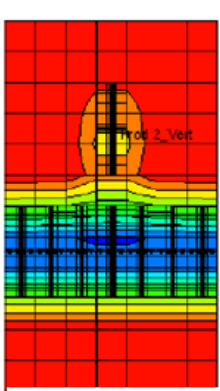

b. $600 \mathrm{ft}$

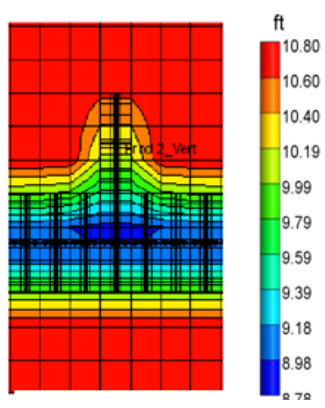

c. $450 \mathrm{ft}$
Figure I 6 Drainage area interference and well spacing for parrallel fractures

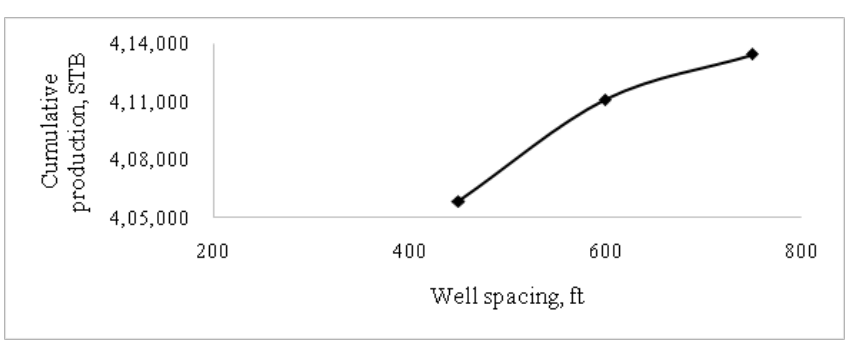

Figure 17 Cumulative production and well spacing

Despite non-visible interference in drainage at wider spacing, having both wells in the same reservoir as far as $750 \mathrm{ft}$ apart shows pressure interference and consequently reduction in volumetric output. Bottom hole pressure (BHP) at the horizontal well remained constant but decreased in the vertical well with closer spacing. Stress field did show no table variation from that seen in Figure15a, for all sampled spacing. Distance of stress orthogonality remained fixed at south of the horizontal well in all cases, but it increased as spacing increased. Away from the horizontal well, this creates an area permissible for transvers fractures with vertical orientation. This advantage can only come into play if both wells are drilled and produced at the same time. This is because the vertical infill well position is too far into the external reservoir where transverse fractures cannot be achieved, instead a horizontal oriented hydraulic fracture will suffice.

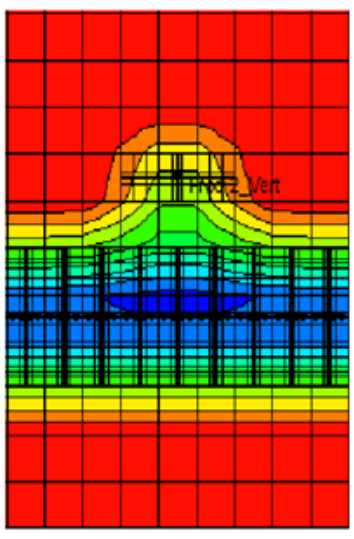

a. $450 \mathrm{ft}$

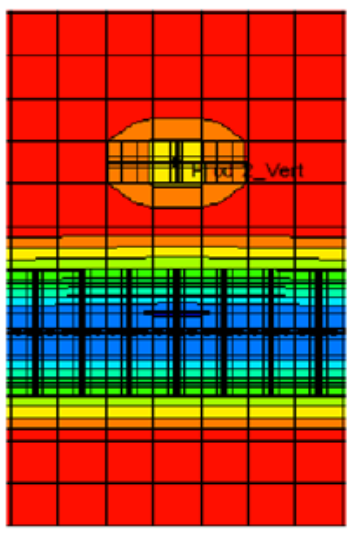

b. $600 \mathrm{ft}$
Figure 18 Drainage area interference and well spacing for perpendicular fractures

\section{Horizontal and vertical wells - perpendicular fracture}

Like parallel vertical fracture model, oil drainage has nodrainage area interference at large spacingat $600 \mathrm{ft}$ and beyond. However, below this, drainage area convergence exists. BHP at the horizontal well was constant for most times but decreases at the vertical well. Also, productivity shows inverse proportionality to well spacing, and the highest output was recorded for the farthest well spacing.

Inference: well spacing - hydraulic fracture orientation - Stress orientation

For both parallel and perpendicular fractures of infill vertical well examined, pressure interference was present in all cases. Larger well spacing resulted in minimum drainage interference and increased production. At such distance, only one of both fracture orientations can be applicable. Usually, infill wells are added at early to mid-period of the field life; 5 years and over. In the model demonstration in a previous section, stress reorientation in horizontal parent well was examined at five years post production in parent well, and minimum distance of orthogonality was approximately $450 \mathrm{ft}$ from the horizontal well axis. Beyond this distance into the reservoir, minimum principal effective stress vector acts vertical. Consequently, only horizontal oriented fracture will propagate perpendicular to the minimum stress direction. Although vertical oriented fracture of infill well at farther spacing is not feasible due to vertical stress orientation. By comparison, at same spacing of $750 \mathrm{ft}$, horizontal oriented fracture gave higher oil production as a justification for the stress direction in the location.

\section{Validation of the model: comparative approach}

Notable well spacing applied in unconventional reservoir models give various values ranging from $330 \mathrm{ft}$ to $1320 \mathrm{ft}$, with a mode spacing value of $700 \mathrm{ft}$ applied in field of practice in Eagle Ford shale. Metrics for the varying distances have been on pressure interference and 
cumulative production only. In this study, a combination of stress distribution and hydrocarbon drainage gives spacing range of $450 \mathrm{ft}$ and $750 \mathrm{ft}$ with $600 \mathrm{ft}$ as minimum recommended. The premises of this inference are in concordance with existing models, whose findings show that pressure transient is a determinant of well spacing and travels beyond SRV into the reservoir for single well. Also, well spacing affects interference and cumulative production inversely.

However, this study offers new insights, particularly in the orientation of infill well and its hydraulic fracture. Table 4 bears these findings.

\section{Conclusion}

Hydrocarbon production in shale reservoir was modelled using coupled geomechanics and fluid flow model. Pressure, drainage volume and stress with respect to time are the main variables considered in this work. Pressure interference models in horizontal and vertical wells were developed as a tool to study well spacing. Using a commercial numerical simulator, drainage volume of a well limited by low permeability formation was delineated, and stress vector profile over the reservoir was obtained. These, including pressure, were used in the well-to-well interference study to examine well spacing and orientation. The following conclusions were drawn:

- Interference effect resulted in reduced cumulative production. Reduction of interference effect is dependent on well spacing; and well spacing is dependent on drainage area of a well, pressure, and distance of stress orthogonality.

- The drainage area of a well stretches beyond stimulated reservoir volume into external reservoir by about $750 \mathrm{ft}$.Locating infill well with drainage area next to a parent well (Zipper pattern) is not economically profitable.

- In-situ stress distribution is uniformly oriented initially but rotates with poroelastic changes in the formation and has tendency to achieve extreme rotation at $90^{\circ}$. Thus, infill wells and their hydraulic fracture cannot have same orientation with parent well.

- Two distances of stress orthogonality $\left(\mathrm{F}_{\sigma}\right)$ exists. One toward the well $\left(\mathrm{F}_{\sigma 1}\right)$, and the other, at further distance into the formation $\left(\mathrm{F}_{\sigma 2}\right)$. In single fracture (vertical well), $\mathrm{F}_{\sigma 1}$ is $0 \mathrm{ft}$, and $\mathrm{F}_{\sigma 2}$ is $750 \mathrm{ft}$. In multiple fractures (horizontal well), $\mathrm{F}_{\sigma 1}$ is $450 \mathrm{ft}$, and $\mathrm{F}_{\sigma 2}$ is unknown (beyond know wides well spacing).

- Separation between $\mathrm{F}_{\sigma} a_{n d F_{\sigma 2}}$ indicates regions where infill well and its hydraulic fractures must be propagated perpendicular to the parent well. Outside this separation, wells and hydraulic fractures parallel to parent well can be achieved.

- Fracture spacing lower than $230 \mathrm{ft}$ should be implemented in very tight reservoir because of increase production recorded. Texas Two Step (Alternate Fracturing) pattern can be employed to achieve this.

- Optimum well spacing for minimum inter-well interference recorded is $600 \mathrm{ft}$

- If horizontal infill-well is considered after productive years, it should be drilled perpendicular to the parent well. This is valid for well spacing of $600 \mathrm{ft}$ and above.

- For irregular reservoir geometry, vertical wells can be drilled, for improved recovery, in locations where horizontal well is not a good fit. The minimum spacing is valid for both horizontal and vertical wells.

\section{Acknowledgment}

The authors acknowledgethe Petroleum Technology Development Fund (PTDF) Nigeria for sponsoring this project. Special thanks to Christie Judith, and members of Computer Modelling Group (CMG) for technical support on the use of CMG-GEM software for this study.

\section{Nomenclature}

$B_{0}$

$c$

Bulk compressibility (1/psi)

$c_{d}$

Total volumetric heat capacity

C

total compressibility, $\left(\mathrm{psi}^{-1}\right)$

E $\quad$ Young's modulus (psi)

$h_{D} \quad$ dimensionless thickness

$h_{f} \quad$ fracture height $(\mathrm{ft})$

$H \quad$ reservoir thickness $(\mathrm{ft})$

$k \quad$ Average permeability (md)

$K_{f} \quad$ hydraulic conductivity

$K_{h} \quad$ bulk thermal conductivity (Btu/(ft $\left.{ }^{\circ} \mathrm{R} \mathrm{d}\right)$

$K_{d r} \quad$ drained bulk modulus (psi)

$K_{r g} \quad$ relative permeability of gas

$K_{r o g} \quad$ relative permeability of oil to gas

$K_{\text {row }} \quad$ relative permeability of oil to water

$K_{r w} \quad$ relative permeability of water

$K \quad \mathrm{x}-\mathrm{y}-\mathrm{z}$-direction permeability respectively

$x, y, z \quad$ (md)

$M \quad$ Biot modulus

$P_{D} \quad$ dimensionless pressure 


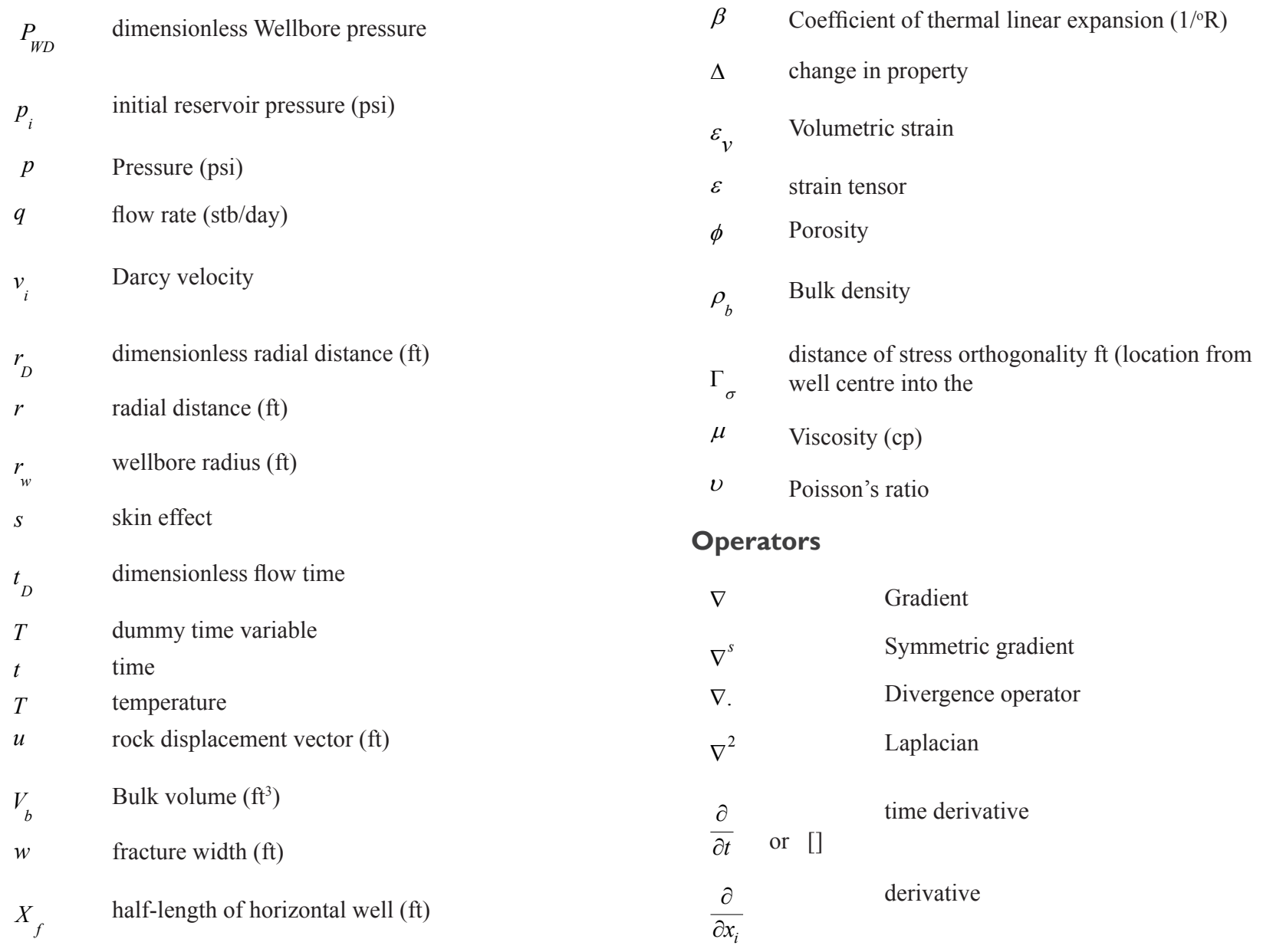

\section{Superscript}

$\begin{array}{ll}n & \text { previous time count } \\ n+1 & \text { current time count }\end{array}$

\section{Appendix A}

Coefficients of porosity: Iterative coupling

$y_{D} \quad$ dimensionless distance in y-direction

$$
\begin{aligned}
& c_{0}=\frac{1}{V_{b}^{0}}\left(\frac{d V_{P}}{d p}+V_{b} \alpha c_{b} \frac{d \sigma_{m}}{d p}-V_{p} \beta \frac{d T}{d p}\right) \\
& c_{1}=-\frac{V_{p}}{V_{b}^{0}} \beta
\end{aligned}
$$

dimensionless well centre in z-direction

\section{Greek letters}

$\alpha \quad$ Biot's poroelastic constant 
$a_{1}=$ factor $\left\{\frac{2}{9} \frac{E}{1-v} \alpha c_{b}\right\}$

$a_{2}=$ factor $\left\{\frac{2}{91-v} \beta\right\}$

\section{Appendix B}

The proposed correlation for multi-rate flow and normalised pressure distribution in the reservoir system is given as follows:

Pressure drop at observation well:

$$
P_{D W}=\int_{0}^{t_{D}} q_{D}\left(\tau_{D}\right) \frac{d P_{D}\left(t_{D}-\tau_{D}\right)}{d \tau_{D}} d \tau_{D}
$$

The corresponding discretised pressure drop is given as:

$$
P_{D W}=\sum_{i=1}^{n}\left(q_{D 1}-q_{D i-1}\right)\left[P_{D}\left(t_{D}-t_{D 1}\right)\right]
$$

where $P_{D}=\left(\frac{P_{i}-P_{i-1}}{q_{n}}\right)$, and $q_{D i}=\frac{q_{i}}{q_{r e f}}, q_{D 0}=0$ at $T_{D 0}=0 ; q_{r e f}$ is fixed reference surface rate.

The dimensionless pressure drop for horizontal well in anisotropic system is:

$P_{D}=\frac{\sqrt{\pi}}{4} \sqrt{\frac{k}{k_{y}}} \int_{0}^{\tau_{D}} \frac{1}{\sqrt{\tau_{D}}} \cdot\left[\exp \left(-\frac{y_{D}^{2}}{4 \tau_{D}}\right)\right]\left[\operatorname{erf} \frac{\left(\sqrt{\frac{k}{k_{x}}}+x_{D}\right)}{2 \sqrt{\tau}}+\operatorname{erf} \frac{\left(\sqrt{\frac{k}{k_{x}}}+x_{D}\right)}{2 \sqrt{\tau}}\right]\left[1+2 \sum_{n-1}^{\infty} \exp \left(\frac{-n^{2} \pi^{2} \tau_{D}}{h_{D}^{2}}\right) \cdot \cos \left(n \pi Z_{w D}\right) \cdot \cos \left(n \pi \frac{Z_{D}}{h_{D}}-Z_{w D}\right)\right] \cdot d \tau{ }_{D}$

where the pressure drop is approximated as;

$$
\begin{aligned}
& P_{D}\left(x_{D}, y_{D}, z_{D}, z_{w D}, h_{D}, \tau\right)=\frac{k h}{141.2 q \mu_{0} \beta_{0}}\left(P_{i}-P\left(x, y, z, z_{w}, h, t\right)\right) \\
& t_{D}=\frac{0.000264 k t}{\phi \mu c_{t}(L / 2)^{2}} \\
& k=3 \sqrt{k_{x} k_{y} k_{z}}
\end{aligned}
$$

In vertical well, dimensionless pressure drop uses the Ei function for continuity equation as:

$$
P_{D}=-\frac{1}{2} E_{i} \frac{-r_{D}^{2}}{4 \tau_{D}}=\frac{k h}{141.2 q \mu \beta}\left(P_{I}-P_{w f}\right)
$$

where, 


$$
t_{D}=\frac{0.000264 k t}{\phi \mu c_{t} r_{i}^{2}}
$$

and $\mathrm{L}$ is the length of the well, Ei approximation is applicable and other dimensionless geometries are defined as follows ${ }^{14}$ :

$$
\begin{aligned}
& X_{D}=\frac{X-X_{W}}{X_{f}} \sqrt{\frac{k}{k_{x}}} \\
& Y_{D}=\frac{Y-Y_{W}}{X_{f}} \sqrt{\frac{k}{k_{y}}} \\
& Z_{D}=\frac{Z-Z_{w}}{X_{f}} \sqrt{\frac{k}{k_{z}}}
\end{aligned}
$$

$$
Z_{W D}=\frac{Z_{w}}{h}
$$$$
h_{D}=\frac{h}{X_{f}} \sqrt{\frac{k}{k_{z}}}
$$

\section{References}

1. Higgins-Borchardt S, Sitchler J, Bratton T. Geomechanics for unconventional reservoirs. In: Unconventional Oil and Gas Resources Handbook. Elsevier; 2016:199-213.

2. Curnow JS, Tutuncu AN. A coupled geomechanics and fluid flow modeling study for hydraulic fracture design and production optimization in an eagle ford shale oil reservoir. In: SPE Hydraulic Fracturing Technology Conference. Society of Petroleum Engineers;

3. Roussel NP, Sharma MM. Optimizing fracture spacing and sequencing in horizontal-well fracturing. SPE Production \& Operations. 2011;26(02):173-184.

4. Rafiee M, Soliman MY, Pirayesh E, Emami Meybodi H. Geomechanical considerations in hydraulic fracturing designs. In: SPE Canadian Unconventional Resources Conference. Society of Petroleum Engineers; 2012.
5. Rafiee M, Soliman MY, Pirayesh E. Hydraulic fracturing design and optimization: a modification to zipper frac. In: SPE Annual Technical Conference and Exhibition. Society of Petroleum Engineers; 2012.

6. Sahai V, Jackson G, Rai RR, Coble L. Optimal well spacing configurations for unconventional gas reservoirs. In: SPE Americas Unconventional Resources Conference. Society of Petroleum Engineers; 2012.

7. Lalehrokh F, Bouma J. Well spacing optimization in eagle Ford. In: SPE/CSUR Unconventional Resources Conference-Canada. Society of Petroleum Engineers; 2014.

8. Charoenwongsa S, Kazemi H, Miskimins J, Fakcharoenphol P. A fully-coupled geomechanics and flow model for hydraulic fracturing and reservoir engineering applications. In: Canadian Unconventional Resources and International Petroleum Conference. Society of Petroleum Engineers; 2010.

9. Zhao X, Ju Y, Yang Y, Su S, Gong W. Impact of hydraulic perforation on fracture initiation and propagation in shale rocks. Science China Technological Sciences. 2016;59(5):756-762.

10. Coussy O. Poromechanics. John Wiley \& Sons; 2004.

11. Fischer-Cripps AC. Introduction to Contact Mechanics. 2nd ed.; 2007.

12. Yang M, Valk PP, Economides MJ. Hydraulic Fracture Production Optimization with a Pseudo-3D Model in Multi-layered Lithology. In: SPE/EAGE European Unconventional Resources Conference \& Exhibition-From Potential to Production. ; 2012.

13. Weng X, Kresse O, Cohen CE, Wu R, Gu H. Modeling of hydraulic fracture network propagation in a naturally fractured formation. In: $S P E$ Hydraulic Fracturing Technology Conference. Society of Petroleum Engineers; 2011.

14. Giegbefumwen PU, Adewole ES. Interference Test Pressures of a Reservoir with Vertical and Horizontal Wells. In: SPE Nigeria Annual International Conference and Exhibition. Society of Petroleum Engineers; 2015.

15. Merzouk K, Tiab D, Escobar FH. Multirate Test in Horizontal Wells In: SPE Asia Pacific Oil and Gas Conference and Exhibition. Society of Petroleum Engineers; 2002.

16. Yu W, Xu Y, Weijermars R, Wu K, Sepehrnoori K. Impact of well interference on shale oil production performance: a numerical model for analyzing pressure response of fracture hits with complex geometries. In: SPE Hydraulic Fracturing Technology Conference and Exhibition. Society of Petroleum Engineers; 2017.

17. Simpson MD, Patterson R, Wu K. Study of Stress Shadow Effects in Eagle Ford Shale: Insight from Field Data Analysis. In: 50th US Rock Mechanics/Geomechanics Symposium. American Rock Mechanics Association; 2016. 